COMMUNICATIONS IN

ANALYSIS AND GEOMETRY

Volume 14, Number 1, 1-24, 2006

\title{
Degeneration of Kähler-Einstein Manifolds II: the Toroidal case
}

\author{
WeI-DONG RUAN ${ }^{1}$
}

\begin{abstract}
In this paper, we prove that the Kähler-Einstein metrics for a toroidal canonical degeneration family of Kähler manifolds with ample canonical bundles Gromov-Hausdorff converge to the complete Kähler-Einstein metric on the smooth part of the central fiber when the base locus of the degeneration family is empty. We also prove the incompleteness of the Weil-Peterson metric in this case.
\end{abstract}

\section{Introduction.}

This paper is a sequel to [8]. In algebraic geometry, when discussing the compactification of the moduli space of complex manifold $X$ with ample canonical bundle $K_{X}$, it is necessary to consider holomorphic degeneration family $\pi: \mathcal{X} \rightarrow B$, where $X_{t}=\pi^{-1}(t)$ are smooth for $t \neq 0, \mathcal{X}$ and $X_{0}$ are $\mathbb{Q}$-Gorenstein, such that the canonical bundle of $X_{t}$ for $t \neq 0$ and the dualizing sheaf of $X_{0}$ are ample. We will call such degeneration canonical degeneration. We are interested in studying the degeneration behavior of the family of Kähler-Einstein metrics $g_{t}$ on $X_{t}$ when $t$ approaches 0. Following his seminal proof of Calabi conjecture [13], Yau [11] initiated the program of studying the application of Kähler-Einstein metrics to algebraic geometry with the belief that the behavior of Kähler-Einstein metrics should reflect the topological, geometric and algebraic structure of the underlying complex algebraic manifolds. According to this philosophy, one would expect the metric degeneration of the Kähler-Einstein manifolds to be closely related to the algebraic degeneration of the underlying algebraic manifolds. In [9], Tian made the first important contribution along this direction. He proved that the Kähler-Einstein metrics on $X_{t}$ converge to the complete Cheng-Yau Kähler-Einstein metric on the smooth part of $X_{0}$ in the sense of CheegerGromov, when $\mathcal{X}$ is smooth and the central fibre $X_{0}$ is the union of smooth normal crossing divisors $D_{1}, \cdots, D_{L}$, with a technical restriction that no

\footnotetext{
${ }^{1}$ Partially supported by NSF Grant DMS-0104150.
} 
three divisors have common intersection. Following the general framework in Tian's paper ([9]), [4] and later [8] studied the general normal crossing case and removed the technical restriction in [9].

In this paper, we generalize the result in [8] to the case when the central fibre $X_{0}$ is a union of toroidal orbifolds that results from the so-called toroidal canonical degeneration of smooth $X_{t}$ (see Section 2 for definitions). The total space $\mathcal{X}$ for this kind of degeneration will be toroidal and generally not smooth. Please note that a toroidal canonical degeneration, where $X_{0}$ is not normal crossing in $\mathcal{X}$, cannot be reduced to a normal crossing canonical degeneration. The normal crossing case is a very special case of toroidal canonical degeneration. For an algebraic curve, a toroidal canonical degeneration is equivalent to Deligne-Mumford stable degeneration into stable curves.

In this paper, we always require an algebraic variety $X$ to possess a (set-theoretical) canonical (Whitney) stratification $X=\bigcup_{p \in \Sigma} D_{p}$ by smooth algebraic strata. By "canonical", we mean that any other (Whitney) stratification $X=\bigcup_{p^{\prime} \in \Sigma^{\prime}} D_{p^{\prime}}^{\prime}$ by smooth strata is a refinement of the canonical (Whitney) stratification. More precisely, we have $D_{p^{\prime}}^{\prime} \subset D_{p}$ when $D_{p^{\prime}}^{\prime} \cap D_{p} \neq \emptyset$. For example, the toroidal varieties defined in Section 2 satisfy such requirement.

The degeneration family is called base point free if each smooth strata of $X_{0}$ is inside a smooth strata of $\mathcal{X}$. The smoothness condition of $\mathcal{X}$ when $X_{0}$ is normal crossing is equivalent to requiring the degeneration family to be base point free. In some sense, toroidal canonical degenerations that we consider in this paper are generic base point free canonical degenerations. (Toroidal canonical degenerations and related concepts and constructions are discussed in Section 2.)

Our first main theorem (proved in Section 5) is the following.

Theorem 1.1. Let $\pi: \mathcal{X} \rightarrow B$ be a toroidal canonical degeneration of Kähler-Einstein manifolds $\left\{X_{t}, g_{E, t}\right\}$ with $\operatorname{Ric}\left(g_{E, t}\right)=-g_{E, t}$. Then, the Kähler-Einstein metrics $g_{E, t}$ on $X_{t}$ converge in the sense of CheegerGromov to a complete Cheng-Yau Kähler-Einstein metric $g_{E, 0}$ on the smooth part of the canonical limit $X_{0}^{\prime}$ (which is a finite cover of the central fibre $X_{0}$ ).

To prove this theorem, we follow the three steps outlined in [8]. The first step is to construct certain smooth family of background Kähler metrics $\hat{g}_{t}$ 
on $X_{t}$ and their Kähler potential volume forms $\hat{V}_{t}$. The second step is to construct a smooth family of approximate Kähler metrics $g_{t}$ with Kähler form $\omega_{t}=\frac{i}{2 \pi} \partial \bar{\partial} \log V_{t}$, where $V_{t}=h \hat{V}_{t}$ ( $h$ is a function on $\left.\mathcal{X}\right)$ satisfies a certain uniform estimate independent of $t$. The third step is to use MongeAmpère estimate of Aubin [1] and Yau [13] to derive a uniform estimate (independent of $t$ ) for the smooth family of Kähler-Einstein metrics $g_{E, t}$, starting with the smooth family of approximate Kähler metric $g_{t}$, which is enough to ensure the Gromov-Hausdorff convergence of the family to the unique complete Kähler-Einstein metric $g_{E, 0}=\left\{g_{0, i}\right\}_{i=1}^{l}$ on the smooth part of $X_{0}$. The first and the third steps are carried out in the very brief Sections 3 and 5 and are virtually the same as in the normal crossing case [8]. The second step, carried out in Section 4, is more involved than the simple global construction in [8].

The following similar but much more non-trivial (comparing to [8]) estimate of the Weil-Peterson metric near the degeneration, which implies the incompleteness of the Weil-Peterson metric, is worked out in Section 6 .

Theorem 1.2. The restriction of the Weil-Peterson metric on the moduli space of complex structures to the toroidal canonical degeneration $\pi: \mathcal{X} \rightarrow B$ is bounded from above by a constant multiple of $\frac{d t \wedge d \bar{t}}{|\log | t||^{3}|t|^{2}}$. In particular, Weil-Peterson metric is incomplete at $t=0$.

Note on notation: We say $A \sim B$ if there exist constants $C_{1}, C_{2}>0$ such that $C_{1} B \leq A \leq C_{2} B$.

\section{Toroidal canonical degeneration.}

In this section, we introduce the concepts of toric degeneration and toroidal degeneration, and discuss the details of relevant stratification structures and the construction of compatible partition functions that we need for the construction of approximate metrics in Section 4.

\subsection{Toric degenerations.}

(Unless specified otherwise, the notations in this subsection will not be carried over to other parts of this paper.)

Let us first introduce the basic notions in toric geometry. An $(n+1)$ dimensional affine toric variety $A_{\sigma_{0}}$ is determined by a strongly convex $(n+1)$-dimensional integral polyhedral cone $\sigma_{0}$ in a rank $n+1$ lattice $\tilde{M}$. 
Let $\sigma_{0}(k)$ denote the set of $k$-dimensional subfaces of $\sigma_{0}$. Then, $\sigma_{0}(n)$ corresponds to toric Weil divisors $\left\{D_{i}\right\}_{i \in \sigma_{0}(n)}$ in $A_{\sigma_{0}}$, and $\sigma_{0}(1)$ corresponds to toric Cartier divisors $\left\{\left(f_{i}\right)\right\}_{i \in \sigma_{0}(1)}$ in $A_{\sigma_{0}}$. For $i \in \sigma_{0}(1)$,

$$
\left(f_{i}\right)=\sum_{j \in \sigma_{0}(n)} a_{i j} D_{j}
$$

where $a_{i j}$ is the natural pairing of the primitive elements in $i \in \sigma_{0}(1)$ and $j \in \sigma_{0}^{\vee}(1) \cong \sigma_{0}(n) . \sigma_{0}^{\vee}$ denotes the dual cone of $\sigma_{0}$.

A toric map $\pi: \mathcal{X} \rightarrow B \cong \mathbb{C}$ is called a toric degeneration, if $\mathcal{X}=A_{\sigma_{0}}$ is an affine toric variety such that $\mathcal{X} \backslash X_{0}$ is the big open torus. Consequently, $X_{t}$ for $t \neq 0$ are codimension one subtori in $\mathcal{X} \backslash X_{0}$. A toric degeneration is determined by a strongly convex integral polyhedral cone $\sigma_{0} \subset \tilde{M}$ with a marked primitive element $t$ in the interior of the cone $\sigma_{0}$. Under such notation, the central fibre is

$$
X_{0}=D=\bigcup_{i \in \sigma_{0}(n)} D_{i} .
$$

Let $M=\tilde{M} / \mathbb{Z}\{t\}$. Since $t$ is in the interior of $\sigma_{0}$, the projection of $\sigma_{0}$ to $M$ determines a complete fan $\Sigma$ on $M$. Splittings $\tilde{M} \cong M \times \mathbb{Z}\{t\}$ can be parameterized (non-canonically) by $\mathbb{Z}$-valued linear functions on $M$. Each such splitting realizes $\sigma_{0}$ as a $\mathbb{Q}$-valued function $w_{\sigma_{0}}$ on the lattice $M$. In such a way, $\sigma_{0}$ can be understood as an equivalence class $\left[w_{\sigma_{0}}\right]$ (modulo $\mathbb{Z}$ valued linear functions) of convex piecewise linear $\mathbb{Q}$-valued functions on the lattice $M$ that are compatible with a complete fan $\Sigma$ in $M$. Let $\Sigma(k)$ denote the set of $k$-dimensional cones in $\Sigma$. Naturally, $\Sigma(k) \cong \sigma_{0}(k)$ for $1 \leq k \leq n$. We will use $\tilde{\sigma} \in \sigma_{0}(k)$ to denote the cone corresponding to $\sigma \in \Sigma(k)$.

For each $\sigma \in \Sigma$, there is an affine variety $A_{\sigma}=\operatorname{Spec}(\mathbb{C}[\sigma])$. For $\sigma, \sigma^{\prime} \in \Sigma$ satisfying $\sigma \subset \sigma^{\prime}$, there is a natural semi-group morphism $\sigma^{\prime} \rightarrow \sigma$ that restricts to identity map on $\sigma \subset \sigma^{\prime}$ and restricts to zero map on $\sigma^{\prime} \backslash \sigma$, which induces the map $h_{\sigma \sigma^{\prime}}: A_{\sigma} \rightarrow A_{\sigma^{\prime}}$. Using $\left\{h_{\sigma \sigma^{\prime}}\right\}_{\sigma, \sigma^{\prime} \in \Sigma}$, we may glue the affine pieces $\left\{A_{\sigma}\right\}_{\sigma \in \Sigma}$ into the singular variety $X_{\Sigma}$. We have the following natural canonical (Whitney) stratification

$$
X_{\Sigma}=\bigcup_{\sigma \in \Sigma} T_{\sigma}, \text { where } T_{\sigma}=\left(\operatorname{Span}_{\mathbb{Z}} \sigma\right)^{\vee} \otimes_{\mathbb{Z}} \mathbb{C}^{*}=\left(M^{\vee} / \sigma^{\perp}\right) \otimes_{\mathbb{Z}} \mathbb{C}^{*} .
$$

In such a way, $\Sigma$ determines a singular variety $X_{\Sigma}$ that is a mirror dual to the usual toric variety $P_{\Sigma}$ in certain sense.

For $\sigma \in \Sigma$, the natural injection $\tilde{\sigma} \hookrightarrow \sigma$ over $\mathbb{Z}$ induces a cover map $p_{\sigma}: A_{\sigma} \rightarrow A_{\tilde{\sigma}}$ and subsequently, $q_{\sigma}=h_{\tilde{\sigma} \sigma_{0}} \circ p_{\sigma}: A_{\sigma} \rightarrow A_{\sigma_{0}}=\mathcal{X}$. It is easy 
to check that $p_{\sigma}, q_{\sigma}$ for $\sigma \in \Sigma$ glue together to form the maps $p_{\Sigma}: X_{\Sigma} \rightarrow X_{0}$, $q_{\Sigma}: X_{\Sigma} \rightarrow \mathcal{X}$

Recall that a complex torus has a canonical toric holomorphic volume form, and consequently, a canonical real toric volume form. Via this toric holomorphic volume form on the complex torus $\mathcal{X} \backslash X_{0}$, the dualizing sheaf $K_{\mathcal{X}}$ can be naturally identified with $\mathcal{O}_{\mathcal{X}}(-D)$. We call $\pi$ simple when each divisor $D_{i}$ is of multiplicity one under $\pi$. Then, the Cartier divisor $(t)=D$, and the dualizing sheaf $K_{\mathcal{X}}$ is a line bundle.

Proposition 2.1. A toric degeneration $\pi: \mathcal{X} \rightarrow B \cong \mathbb{C}$ is simple if and only if $\left[w_{\sigma_{0}}\right]$ is $\mathbb{Z}$-valued on $M$ if and only if $q_{\Sigma}: X_{\Sigma} \rightarrow \mathcal{X}$ is an imbedding (or equivalently, $p_{\Sigma}: X_{\Sigma} \rightarrow X_{0}$ is an isomorphism).

Proof. For $\tilde{\sigma} \in \sigma_{0}(n)$, it is straightforward to check that the multiplicity of $t$ along $D_{\tilde{\sigma}}$ is $\left|\left(\operatorname{Span}_{\mathbb{Z}} \sigma\right) /\left(\operatorname{Span}_{\mathbb{Z}} \tilde{\sigma}\right)\right|$. Consequently, $\pi$ is simple if and only if for each $\sigma \in \Sigma(n)$, the natural injection $\tilde{\sigma} \hookrightarrow \sigma$ over $\mathbb{Z}$ is bijection (which amounts to that $\left[w_{\sigma_{0}}\right]$ is $\mathbb{Z}$-valued on $\sigma$ ) if and only if $p_{\sigma}: A_{\sigma} \rightarrow A_{\tilde{\sigma}}$ is an isomorphism for each $\sigma \in \Sigma(n)$. These local results together imply the proposition.

Proposition 2.2. For a toric degeneration $\pi: \mathcal{X} \rightarrow B \cong \mathbb{C}$, let $d$ be the smallest positive integer so that $d\left[w_{\sigma_{0}}\right]$ is $\mathbb{Z}$-valued. Then, the canonical $d^{\prime}$ fold base extension $\pi^{\prime}: \mathcal{X}^{\prime} \rightarrow B^{\prime}$ is a simple toric degeneration if and only if $d \mid d^{\prime}$.

Proof. It is easy to see that the canonical $d^{\prime}$-fold base extension $\pi^{\prime}: \mathcal{X}^{\prime} \rightarrow B^{\prime}$ is determined by $d^{\prime} \sigma_{0} \subset \tilde{M}$. Since $\left[w_{d^{\prime} \sigma_{0}}\right]=d^{\prime}\left[w_{\sigma_{0}}\right]$ is $\mathbb{Z}$-valued if and only if $d \mid d^{\prime}$, by Proposition 2.1, we get the desired conclusion.

Remark: Propositions 2.1 and 2.2 are well known. (A special case of Proposition 2.2, where $\Sigma$ is simplicial fan, was proved and used in the proof of the semistable reduction theorem [5] by Mumford and Kun.) We provide simple proofs of them here for the convenience of the readers.

$\Sigma(1)$ can be equivalently interpreted as the set of primitive generating elements of 1-dimensional cones in $\Sigma$. The piecewise linear function $w_{\sigma_{0}}$ is determined by $\left\{w_{m}\right\}_{m \in \Sigma(1)}$, with $w_{m}=w_{\sigma_{0}}(m) \in \mathbb{Q}$ for $m \in \Sigma(1)$. The toric degeneration family can be equivalently characterized by the following 
family of toric immersions:

$$
i_{t}: N_{\mathbb{C}^{*}} \rightarrow \mathbb{C}^{|\Sigma(1)|}
$$

defined as $\left\{t^{w_{m}} z^{m}\right\}_{m \in \Sigma(1)}$, where $N=M^{\vee}$ and $N_{\mathbb{C}^{*}}=\left(N \otimes_{\mathbb{Z}} \mathbb{C}\right) / N$. We are also interested in generalized toric degenerations, where $w_{m} \in \mathbb{R}$ are not necessarily rational.

Example: The simplest toric degenerations that are not normal crossing are:

(1) $X_{t}=\left\{z \in \mathbb{C}^{4} \mid z_{1} z_{2}=z_{3} z_{4}=t\right\}$ (product of normal crossing degenerations).

(2) $X_{t}=\left\{z \in \mathbb{C}^{4} \mid z_{1} z_{2}=t, z_{3} z_{4}=t z_{1}\right\}$.

Remark: A priori, the piecewise linear function $f$ generated by $\left\{w_{m}\right\}_{m \in \Sigma(1)}$ need not be convex. Then, we may take the largest convex function $\tilde{f} \leq f$. The piecewise linear convex function $\tilde{f}$ will be generated by $\left\{w_{m}\right\}_{m \in \tilde{\Sigma}(1)}$, where $\tilde{\Sigma}(1)$ is a subset of $\Sigma(1)$. There is a natural projection $P: \mathbb{C}^{|\Sigma(1)|} \rightarrow$ $\mathbb{C}^{|\tilde{\Sigma}(1)|}$. It is easy to check that $P$ induces an equivalence between the toric degeneration families determined by toric embeddings $i_{t}$ and $\tilde{i}_{t}=P \circ i_{t}$. Therefore, we only need to consider the case when $f$ is convex. For $f$ generic, the fan it determines is a simplicial fan. Namely, the toric divisors are all toric orbifolds.

\subsection{Toroidal degenerations.}

A holomorphic degeneration $\pi: \mathcal{X} \rightarrow B=\{t \in \mathbb{C}:|t|<1\}$ is called a toroidal degeneration if it is locally toric. Let

$$
X_{0}=\bigcup_{k=0}^{n} X_{0}^{(k)}=D=\bigcup_{p \in \Sigma} D_{p}, X_{0}^{(k)}=\bigcup_{p \in \Sigma(k)} D_{p}, \Sigma=\bigcup_{k=0}^{n} \Sigma(k)
$$

be the canonical stratification for $X_{0}$, with $\left\{D_{p}\right\}_{p \in \Sigma}$ parameterizing all the strata and $X_{0}^{(k)}$ denoting the union of all $k$-dimensional strata. $\pi$ is called simple if each divisor $\bar{D}_{p}$ is of multiplicity 1 under $\pi$ for $p \in \Sigma(n)$. Propositions 2.1 and 2.2 imply the following generalization to toroidal case.

Proposition 2.3. For a toroidal degeneration $\pi: \mathcal{X} \rightarrow B$, there exists an integer $d>0$ such that the canonical $d^{\prime}$-fold base extension $\pi^{\prime}: \mathcal{X}^{\prime} \rightarrow B^{\prime}$ is a simple toroidal degeneration if and only if $d \mid d^{\prime} . X_{0}^{\prime}$ (which will be called the canonical limit) is independent of $d^{\prime}$ satisfying $d \mid d^{\prime}$ with the natural finite cover map $X_{0}^{\prime} \rightarrow X_{0}$. 
Proof. Since $d^{\prime}$-fold base extension is canonical and local, the $d>0$ here can be taken to be the lowest common multiple of the $d$ 's specified in Proposition 2.2 for all local toric models. When $d \mid d^{\prime}$, namely $\pi^{\prime}$ is simple, Proposition 2.1 implies that $X_{0}^{\prime}$ restricted to each local toric model can be identified with $X_{\Sigma}$ in Proposition 2.1, therefore is canonical and independent of $d^{\prime}$.

Through Proposition 2.3, all discussions for toroidal degeneration can be reduced to discussions for simple toroidal degeneration via base extension. For this reason, we will always assume that $\pi$ is simple. Consequently, the dualizing sheaf $K_{\mathcal{X}}$ is a line bundle. For such generic degeneration $\pi$, the Weil divisors $\bar{D}_{p}$ for $p \in \Sigma(n)$ are toroidal orbifolds. Without loss of generality and for simplicity of notations, we will also assume that each $\bar{D}_{p}$ does not self-intersect.

Choose a suitable tubular neighborhood $\tilde{U}_{p}$ of $\bar{D}_{p}$ for each $p \in \Sigma$ such that for any $p_{1}, p_{2} \in \Sigma$, we have

$$
\tilde{U}_{p_{1}} \cap \tilde{U}_{p_{2}} \subset \bigcup_{q \in \Sigma, D_{q} \in \bar{D}_{p_{1}} \cap \bar{D}_{p_{2}}} \tilde{U}_{q} .
$$

For each $p \in \Sigma$, we can construct a tubular neighborhood $U_{p}$ of $D_{p}$ as $\tilde{U}_{p}$ minus the union of divisors $\bar{D}_{q}$ for $q \in \Sigma(n)$ satisfying $D_{p} \not \subset \bar{D}_{q}$. We will also need $U_{p}^{0} \subset U_{p}$ defined as $\tilde{U}_{p}$ minus the union of (slightly shrunk) $\overline{\tilde{U}}_{q}$ for $q \in \Sigma(n)$ satisfying $D_{p} \not \subset \bar{D}_{q}$. Let $D_{p}^{0}=D_{p} \cap U_{p}^{0}$. Since

$$
\bigcup_{p \in \Sigma} U_{p}=\bigcup_{p \in \Sigma} U_{p}^{0}
$$

forms a neighborhood of $X_{0}$ that contains $X_{t}$ for $t$ small, many of our discussions on $X_{t}$ can be reduced locally to either $U_{p} \cap X_{t}$ or $U_{p}^{0} \cap X_{t}$ for $p \in \Sigma$. Notice that for any $p_{1}, p_{2} \in \Sigma$, we also have

$$
U_{p_{1}} \cap U_{p_{2}} \subset \bigcup_{q \in \Sigma, D_{q} \in \bar{D}_{p_{1}} \cap \bar{D}_{p_{2}}} U_{q}, \quad U_{p_{1}}^{0} \cap U_{p_{2}}^{0} \subset \bigcup_{q \in \Sigma, D_{q} \in \bar{D}_{p_{1}} \cap \bar{D}_{p_{2}}} U_{q}^{0} .
$$

Locally, $U_{p}=A_{p} \times D_{p}$ and $U_{p}^{0}=A_{p} \times D_{p}^{0} . A_{p}$ is a neighborhood of the origin of the affine toric local model determined by the fan $\Sigma_{p}$ and the integral convex function $\left\{w_{m}\right\}_{m \in \Sigma_{p}(1)}$ (notation as in 2.1). Let $|p|:=\operatorname{dim} D_{p}$ and $l=n-|p| . \quad \Sigma_{p}(l)$ (which can be naturally identified with a subset of $\Sigma(n))$ corresponds to toroidal Weil divisors $\left\{D_{q} \cap U_{p}\right\}_{q \in \Sigma_{p}(l) \subset \Sigma(n)}$ in $U_{p}$. $\Sigma_{p}(1)$ corresponds to toroidal Cartier divisors $\left\{\left(s_{m}\right)\right\}_{m \in \Sigma_{p}(1)}$ in $U_{p}$ containing $D_{p}$. We may choose local coordinate $(t, z, \tilde{z})$ for $U_{p}, z=\left(z_{1}, \cdots, z_{l}\right)$, 
$\tilde{z}=\left(z_{l+1}, \cdots, z_{n}\right)$, so that $s_{m}=t^{w_{m}} z^{m},(t, z)$ and $\tilde{z}$ form coordinates for $A_{p}$ and $D_{p}$. $(z, \tilde{z})$ can be considered as coordinate for $X_{t} \cap U_{p}$. For $m \in \Sigma_{p}(1)$, $s_{m}$ can be viewed as a section of a line bundle on $U_{p}$ that defines the Cartier divisor. One can choose a Hermitian metric $\|\cdot\|_{m}$ on the line bundle over $U_{p}^{0}$ such that $\left\|s_{m}\right\|_{m} \leq 1$ and $\left\|s_{m}\right\|_{m}=1$ outside a small neighborhood of the Cartier divisor $\left(s_{m}\right)$. More precisely, we require that $\left\|s_{m}\right\|_{m}=1$ on $U_{q}^{0}$ for $q \in \Sigma$ when $s_{m}$ is non-vanishing on $D_{q}$.

For $p, q \in \Sigma$ satisfying $D_{q} \subset \bar{D}_{p}$, Cartier divisors in $U_{p}$ can be naturally extended to certain $\mathbb{Q}$-Cartier divisors in $U_{q}$ that can be expressed by the natural injective map $e_{p q}: \Sigma_{p}(1) \rightarrow \Sigma_{q}(1)$. By suitably adjusting the Hermitian metric of the line bundle, for $m \in \Sigma_{p}(1)$, we may assume that $\left\|s_{m}\right\|_{m}=\left\|s_{e_{p q}(m)}\right\|_{e_{p q}(m)}$ in the common domain $U_{p}^{0} \cap U_{q}^{0}$. It is easy to check that $e_{p q^{\prime}}=e_{q q^{\prime}} \circ e_{p q}$ for $q^{\prime} \in \Sigma$ satisfying $D_{q^{\prime}} \subset \vec{D}_{q}$. Therefore, the Cartier divisor $\left(s_{m}\right)$ in $U_{p}^{0}$ for $m \in \Sigma_{p}(1)$ naturally extends to the $\mathbb{Q}$-Cartier divisor (still denoted by $\left.\left(s_{m}\right)\right)$ in $\tilde{U}_{p}$. $\left\|s_{m}\right\|_{m}$ for $m \in \Sigma_{p}(1)$ can similarly be extended from $U_{p}^{0}$ to $\tilde{U}_{p}$.

Let $\Sigma^{p}(1)$ denote the set of $q \in \Sigma(|p|+1)$ satisfying $D_{q} \subset \bar{D}_{p}$. For $q \in \Sigma^{p}(1), D_{p}$ can be naturally identified with an element $\left[D_{p}\right] \in \Sigma_{q}(|q|-$ $|p|)=\Sigma_{q}(1)$, which can also be viewed as a Cartier divisor $s_{q}$ in $\tilde{U}_{p}$ supported in $\tilde{U}_{p} \backslash U_{p} . \Sigma_{p}^{p}=\Sigma_{p}(1) \cup \Sigma^{p}(1)$ (resp. $\left.\Sigma^{p}(1)\right)$ can be characterized as the set of Cartier divisors on $\tilde{U}_{p}$ whose defining functions are not identically zero (resp. nowhere zero) on $X_{0} \cap U_{p}$.

A (holomorphic) volume form on $U_{p} \backslash D$ is called toroidal if its pullback to the local toric model differs from the standard toric (holomorphic) volume form by a bounded nowhere zero (holomorphic) factor on $U_{p}$. By examining the holomorphic toric volume form, it is easy to see that a holomorphic toroidal volume form on $\mathcal{X} \backslash D$ can be naturally identified with a nowhere zero holomorphic section of $K_{\mathcal{X}}(D)$, or in another word, a meromorphic section of $K_{\mathcal{X}}$ with a pole of order 1 along $D$.

\subsection{Partition functions.}

Let $\mu(x)$ be a smooth increasing function on $\mathbb{R}$ with bounded derivatives satisfying $\mu(x)=0$ for $x \leq 0$ and $\mu(x)=1$ for $x \geq 1$. Let $\min ^{\prime}\left(x_{1}, \cdots, x_{l}\right)$ be a smooth function with bounded derivatives that coincide with $\min \left(x_{1}, \cdots, x_{l}\right)$ when $\min _{i \neq j}\left(\left|x_{i}-x_{j}\right|\right) \geq 1$. (In another word, $\min ^{\prime}\left(x_{1}, \cdots, x_{l}\right)$ is a smoothing of $\min \left(x_{1}, \cdots, x_{l}\right)$ with bounded derivatives.) 
For each $p \in \Sigma$ and $\eta>0$ large, we may define the smooth function

$$
\tilde{\mu}_{p}=\mu\left(\frac{1}{\log \left(\tau / \eta^{2}\right)} \min ^{\prime}\left(\left\{\log \left(a_{m} / \eta\right)\right\}_{m \in \Sigma_{p}(1)},\left\{\log \left(\tau / a_{m} \eta\right)\right\}_{m \in \Sigma^{p}(1)}\right)\right),
$$

where $\tau=-\log |t|^{2}$ and $a_{m}=\eta-\log \left\|s_{m}\right\|_{m}^{2}$. These will give us the partition functions $\left\{\mu_{p}\right\}_{p \in \Sigma}$, where $\mu_{p}=\tilde{\mu}_{p}\left(\sum_{p \in \Sigma} \tilde{\mu}_{p}\right)^{-1}$. We generally have $D_{p}^{0} \subset$ $\operatorname{supp}\left(\mu_{p}\right) \subset U_{p}$. The condition on $\|\cdot\|_{m}$ implies that

$$
U_{p}^{0} \cap \operatorname{supp}\left(\mu_{q}\right)=\emptyset \text { when } D_{p} \not \subset \bar{D}_{q} .
$$

\section{Construction of the background metric.}

For construction in this section to work, it is necessary to assume that the dualizing line bundle $K_{\mathcal{X}}$ of the total space $\mathcal{X}$ exists and is ample, which is valid in our situation. (The construction in this section is partially inspired by our work [7] on Bergmann metrics.) Recall that $K_{\mathcal{X} / B}=K_{\mathcal{X}} \otimes K_{B}^{-1}$ and $K_{X_{t}}=\left.\left.K_{\mathcal{X} / B}\right|_{X_{t}} \cong K_{\mathcal{X}}\right|_{X_{t}}$. (The last equivalence is not canonical, depending on the trivialization $K_{B} \cong \mathcal{O}_{B}$. We will use $d t$ to fix the trivialization of $K_{B}$.) Since $K_{X_{t}}$ is ample for all $t$, certain multiple $K_{X_{t}}^{m}$ will be very ample for all $t$. Equivalently, $K_{\mathcal{X}}^{m}$ is very ample on $\mathcal{X}$. It is not hard to find sections $\left\{\Omega_{k}\right\}_{k=0}^{N_{m}}$ of $K_{\mathcal{X}}^{m}$ that determine an embedding $e: \mathcal{X} \rightarrow \mathbb{C P}^{N_{m}}$, such that $\left\{\Omega_{t, k}\right\}_{k=0}^{N_{m}}$ forms a basis of $H^{0}\left(K_{X_{t}}\right)$ for all $t$, where $\Omega_{t, k}=\left.\left(\Omega_{k} \otimes(d t)^{-m}\right)\right|_{X_{t}}$. $\left\{\Omega_{t, k}\right\}_{k=0}^{N_{m}}$ will determine a family of embedding $e_{t}: X_{t} \rightarrow \mathbb{C P}^{N_{m}}$ such that $e_{t}=\left.e\right|_{X_{t}}$. Choose the Fubini-Study metric $\omega_{F S}$ on $\mathbb{C P}^{N_{m}}$, and define

$$
\hat{\omega}=\frac{1}{m} e^{*} \omega_{F S}, \quad \hat{\omega}_{t}=\left.\hat{\omega}\right|_{X_{t}}=\frac{1}{m} e_{t}^{*} \omega_{F S} .
$$

Since $K_{\mathcal{X}}^{m}$ is very ample on $\mathcal{X}, \hat{\omega}$ is a smooth metric on $\mathcal{X}$. The Kähler potential of $\hat{\omega}$ and $\hat{\omega}_{t}$ are the logarithm of the volume forms

$\hat{V}=\left(\sum_{k=0}^{N_{m}} \Omega_{k} \otimes \bar{\Omega}_{k}\right)^{\frac{1}{m}}$, and $\hat{V}_{t}=\left(\sum_{k=0}^{N_{m}} \Omega_{t, k} \otimes \bar{\Omega}_{t, k}\right)^{\frac{1}{m}}=\left.\hat{V} \otimes(d t \otimes d \bar{t})^{-1}\right|_{X_{t}}$.

Since $K_{\mathcal{X}}^{m}$ is ample and therefore base point free, $\hat{V}$ is a non-degenerate smooth volume form on $\mathcal{X}$. Recall $(t)=D$. Hence, $\frac{\hat{V}}{|t|^{2}}$ is a toroidal volume form on $\mathcal{X}$. On the other hand, $\frac{d t}{t}$ is the standard toric holomorphic form 
on $B$. Therefore,

$$
\hat{V}_{t}=\left(\sum_{k=0}^{N_{m}} \Omega_{t, k} \otimes \bar{\Omega}_{t, k}\right)^{\frac{1}{m}}=\left.\hat{V} \otimes(d t \otimes d \bar{t})^{-1}\right|_{X_{t}}=\left.\frac{\hat{V}}{|t|^{2}} \otimes\left(\frac{d t}{t} \otimes \frac{d \bar{t}}{\bar{t}}\right)^{-1}\right|_{X_{t}}
$$

is also toroidal, namely

$$
\hat{V}_{t}=\rho(t, z, \tilde{z})\left(\prod_{j=1}^{l} \frac{d z_{j} d \bar{z}_{j}}{\left|z_{j}\right|^{2}}\right)\left(\prod_{j=l+1}^{n} d z_{j} d \bar{z}_{j}\right)
$$

under the coordinate $(z, \tilde{z})$ for $X_{t} \cap U_{p}$, where $\rho(t, z, \tilde{z}) \sim 1$ is a smooth positive function on $U_{p}$.

Since $e: \mathcal{X} \rightarrow \mathbb{C P}^{N_{m}}$ is an embedding, locally in $U_{p}$, there exists a decomposition $e=\hat{e} \circ i_{\Sigma_{p}}$, where $i_{\Sigma_{p}}=\left(s_{\Sigma_{p}}, \tilde{z}\right): \mathcal{X} \rightarrow \mathbb{C}^{\left|\Sigma_{p}(1)\right|+|p|}$ and $\hat{e}: \mathbb{C}^{\left|\Sigma_{p}(1)\right|+|p|} \rightarrow \mathbb{C P}^{N_{m}}$ are smooth embeddings and $s_{\Sigma_{p}}=\left(s_{m}\right)_{m \in \Sigma_{p}(1)}$. Therefore,

$$
\hat{\omega}=\sum_{m, m^{\prime} \in \Sigma_{p}(1)} g_{m m^{\prime}}\left(s_{\Sigma_{p}}, \tilde{z}\right) d s_{m} d \bar{s}_{m^{\prime}}+(\text { terms involving } d \tilde{z}, d \overline{\tilde{z}})
$$

\section{Construction of the approximate metric.}

The approximate metric is constructed by gluing together appropriate metrics on the neighborhood of each strata by the partition functions constructed in Section 2.3.

For $p \in \Sigma$ and $m \in \Sigma_{p}^{p}$, in $U_{p}$, define

$$
h_{p}=\tau^{2\left(\left|\Sigma_{p}(1)\right|-l\right)} \prod_{m \in \Sigma_{p}^{p}} \frac{\eta^{2}}{a_{m}^{2}}, \quad a_{m}=\eta-\log \left\|s_{m}\right\|_{m}^{2}, \tau=-\log |t|^{2} .
$$

On $X_{t}$, let $V_{t}=h \hat{V}_{t}$, where $\log h=\sum_{p \in \Sigma} \mu_{p} \log h_{p}$, and let

$$
\omega_{t}=\frac{i}{2 \pi} \partial \bar{\partial} \log V_{t}=\hat{\omega}_{t}+\frac{i}{2 \pi} \partial \bar{\partial} \log h=\hat{\omega}_{t}+\gamma_{t}+\alpha_{t}
$$


where

$$
\begin{aligned}
\alpha_{t}= & \sum_{p \in \Sigma} \mu_{p} \alpha_{t, p}, \quad \alpha_{t, p}=\frac{i}{\pi} \sum_{m \in \Sigma_{p}^{p}} \frac{1}{a_{m}^{2}} \partial a_{m} \bar{\partial} a_{m}, \\
\gamma_{t}= & \sum_{p \in \Sigma} \mu_{p} \sum_{m \in \Sigma_{p}(1)} \frac{2}{a_{m}} \operatorname{Ric}\left(\|\cdot\|_{m}\right) \\
& +\frac{i}{2 \pi} \sum_{p \in \Sigma}\left(\log h_{p} \partial \bar{\partial} \mu_{p}+\partial \log h_{p} \bar{\partial} \mu_{p}+\partial \mu_{p} \bar{\partial} \log h_{p}\right) .
\end{aligned}
$$

The main result of this section is the estimate (Propositions 4.6 and 4.7) on the approximate Kähler metric $g_{t}$ with the Kähler form $\omega_{t}$ on $X_{t}$.

Since $\Sigma_{p}$ is a simplicial fan, $\sigma \in \Sigma_{p}(l)$ naturally corresponds to a subset $S_{\sigma} \subset \Sigma_{p}(1)$ with $l$ elements.

Proposition 4.1. There exist $\lambda_{1}, \lambda_{2}>0$ such that $\log \left\|s_{m}\right\|_{m}^{2} \geq \lambda_{2} \log |t|^{2}$ on $U_{p}^{0}$ for all $m \in \Sigma_{p}(1)$. And for any $x \in U_{p}^{0}, S_{x}=\{m \in$ $\left.\left.\Sigma_{p}(1)\left|\log \left\|s_{m}(x)\right\|^{2} \geq \lambda_{1} \log \right| t\right|^{2}\right\} \subset S_{\sigma}$ for some $\sigma \in \Sigma_{p}(l)$.

Proof. Since $\log \left\|s_{m}\right\|_{m}^{2}=\log \left|s_{m}\right|^{2}+O(1)$, where $\left|s_{m}\right|$ is the absolute value of $s_{m}$ viewed as monomial in the toric local model, it is sufficient to prove the proposition for $\log \left|s_{m}\right|^{2}$ in the place of $\log \left\|s_{m}\right\|_{m}^{2}$. For $m \in \Sigma_{p}(1)$, there exists $\sigma \in \Sigma_{p}(l)$ such that $-m$ belongs to the cone spanned by $S_{\sigma}$. Namely,

$$
m=-\sum_{m^{\prime} \in S_{\sigma}} b_{m^{\prime}} m^{\prime}
$$

where $b_{m^{\prime}} \geq 0$ for all $m^{\prime} \in S_{\sigma}$. Therefore,

$$
\begin{aligned}
\log \left|s_{m}\right|^{2}= & w_{m} \log |t|^{2}+\log \left|z^{m}\right|^{2}=w_{m} \log |t|^{2}-\sum_{m^{\prime} \in S_{\sigma}} b_{m^{\prime}} \log \left|z^{m^{\prime}}\right|^{2} \\
= & \left(w_{m}+\sum_{m^{\prime} \in S_{\sigma}} b_{m^{\prime}} w_{m^{\prime}}\right) \log |t|^{2}-\sum_{m^{\prime} \in S_{\sigma}} b_{m^{\prime}} \log \left|s_{m^{\prime}}\right|^{2} \\
& \geq\left(w_{m}+\sum_{m^{\prime} \in S_{\sigma}} b_{m^{\prime}} w_{m^{\prime}}\right) \log |t|^{2} .
\end{aligned}
$$

We may take $\lambda_{2}$ to be the maximum of such $\left(w_{m}+\sum_{m^{\prime} \in S_{\sigma}} b_{m^{\prime}} w_{m^{\prime}}\right)$.

Take a subset $S^{\prime} \subset S$ such that $S^{\prime}$ span a simplicial cone and $S^{\prime} \not \subset S_{\sigma}$ for any $\sigma \in \Sigma_{p}(l)$. There exists a linear function $v_{m}$ on $M$ such that $v_{m}=$ 
$\frac{\log \left|s_{m}\right|^{2}}{\log |t|^{2}} \leq \lambda_{1}$ for $m \in S^{\prime}$ and $\left|v_{m}\right| \leq C \lambda_{1}$ for $m \in \Sigma_{p}(1) \backslash S^{\prime}$. Then,

$$
w_{m}^{\prime}=\frac{\log \left|s_{m}\right|^{2}}{\log |t|^{2}}-v_{m}=w_{m}+\frac{\log \left|z^{m}\right|^{2}}{\log |t|^{2}}-v_{m}
$$

is an adjustment of $w_{m}$ by a linear function on $M$, such that $w_{m}^{\prime}=0$ for $m \in S^{\prime}$. Since $S^{\prime} \not \subset S_{\sigma}$ for any $\sigma \in \Sigma_{p}(l)$. The strict convexity of $\left\{w_{m}\right\}_{m \in \Sigma_{p}(1)}$ implies that there exists an $m^{\prime} \in \Sigma_{p}(1) \backslash S^{\prime}$ such that $w_{m^{\prime}}^{\prime}<0$ is the smallest. Take $\lambda_{3}$ to be the maximum of such $w_{m^{\prime}}^{\prime}<0$ for all possible $S^{\prime}$, which have only finite many possibilities. Then, $\lambda_{3}<0$ and

$$
\frac{\log \left|s_{m^{\prime}}\right|^{2}}{\log |t|^{2}}=w_{m^{\prime}}^{\prime}+v_{m^{\prime}} \leq \lambda_{3}+C \lambda_{1}
$$

We may take $\lambda_{1}>0$ to be small so that $\lambda_{3}+C \lambda_{1}<0$. Then, $\left|s_{m^{\prime}}\right|^{2}$ has to be big, contradicting the fact that $\left|s_{m^{\prime}}\right|^{2}$ is small in $U_{p}$. Therefore, $S \subset S_{\sigma}$ for some $\sigma \in \Sigma_{p}(l)$.

\section{Lemma 4.2.}

$$
\gamma_{t}=O\left(\hat{\omega}_{t} / \eta\right)+O\left(\left(\hat{\omega}_{t}+\alpha_{t}\right) / \log \tau\right), \text { where } \tau=-\log |t|^{2} .
$$

Proof. In the argument of this paper, we will always first fix $\eta>0$ large and then take $\tau$ large according to the fixed $\eta$. By our construction, $a_{m} \geq \eta$ is large. Hence,

$$
\sum_{p \in \Sigma} \mu_{p} \sum_{m \in \Sigma_{p}(1)} \frac{2}{a_{m}} \operatorname{Ric}\left(\|\cdot\|_{m}\right)=O\left(\hat{\omega}_{t} / \eta\right) .
$$

For any $x \in X_{t}$, there exist a $q \in \Sigma$ such that $x \in X_{t} \cap U_{q}^{0}$. Since

$$
\sum_{p \in \Sigma} \mu_{p}=1, \quad \sum_{p \in \Sigma} \partial \bar{\partial} \mu_{p}=0
$$

We have

$$
\sum_{p \in \Sigma} \log h_{p} \partial \bar{\partial} \mu_{p}=\sum_{p \in \Sigma}\left(\log h_{p}-\log h_{q}\right) \partial \bar{\partial} \mu_{p}
$$

Since $U_{q}^{0} \cap \operatorname{supp}\left(\mu_{p}\right)=\emptyset$ when $D_{q} \not \subset \bar{D}_{p}$ according to (2.3), we may consider only those $p \in \Sigma$ satisfying $D_{q} \subset \bar{D}_{p}$. Then, there are the natural inclusions $\Sigma^{q}(1) \subset \Sigma^{p}(1), \Sigma_{p}(1) \subset \Sigma_{q}(1)$ and the Cartier divisors in $\Sigma^{p}(1) \backslash \Sigma^{q}(1)$ 
vanishing along $D_{q}$ can be naturally identified with a subset of $\Sigma_{p}(1) \backslash$ $\Sigma_{q}(1)$. Under such identifications $\Sigma_{q}^{q} \cap \Sigma_{p}^{p}$ is defined. For any $m \in \Sigma_{p}^{p} \backslash \Sigma_{q}^{q}$, $\left(s_{m}\right) \cap \bar{D}_{q}=\emptyset$. Consequently, $\left\|s_{m}\right\|_{m}^{2}=1$ and $a_{m}=\eta$ on $\tilde{U}_{q}$ for $m \in \Sigma_{p}^{p} \backslash \Sigma_{q}^{q}$. Hence,

$$
\log h_{p}-\log h_{q}=2 \sum_{m \in \Sigma_{q}^{q} \backslash \Sigma_{p}^{p}} \log \frac{a_{m}}{\tau \eta}
$$

is bounded on $\operatorname{supp}\left(\mu_{p}\right) \cap U_{q} \subset U_{p} \cap U_{q}$. From the explicit expressions of $\mu_{p}$ and $h_{p}$, it is straightforward to check that $\partial \bar{\partial} \mu_{p}=O(1 / \log \tau), \partial \log h_{p}=$ $O(1)$ and $\partial \mu_{p}=O(1 / \log \tau)$ with respect to the Hermitian metric $\hat{\omega}_{t}+\alpha_{t}$. (Such kind of verification is more carefully done in the proof of Proposition 4.5 using (4.1).) Consequently, $\gamma_{t}=O\left(\hat{\omega}_{t} / \eta\right)+O\left(\left(\hat{\omega}_{t}+\alpha_{t}\right) / \log \tau\right)$.

$$
\begin{aligned}
& \text { For } \sigma \in \Sigma_{p}(l) \text {, let } A_{\sigma}(x)=\min _{m \in \Sigma_{p}(1) \backslash S_{\sigma}} a_{m}(x) \text { and } \\
& \qquad U_{p \sigma}^{0}=U_{p \sigma} \cap U_{p}^{0}, U_{p \sigma}=\left\{x \in U_{p} \mid A_{\sigma}(x) \geq A_{\sigma^{\prime}}(x) \text { for } \sigma^{\prime} \in \Sigma_{p}(l)\right\} .
\end{aligned}
$$

Then, the Proposition 4.1 implies that $A_{\sigma}(x) \geq \lambda_{1} \tau>0$ for $x \in U_{p \sigma}^{0}$ and

Proposition 4.3. For $t$ small enough, we have

$$
X_{t} \cap U_{p}^{0}=\bigcup_{\sigma \in \Sigma_{p}(l)} X_{t} \cap U_{p \sigma}^{0},
$$

and $a_{m}^{2} \sim\left(\log |t|^{2}\right)^{2}$ in $U_{p \sigma}^{0}$ for $m \in \Sigma(1) \backslash S_{\sigma}$.

For $S_{\sigma}=\left\{m_{1}, \cdots, m_{l}\right\}$, on $U_{p \sigma}$, we may choose coordinate $z=\left\{z_{k}\right\}_{k=1}^{l}=$ $\left\{s_{m_{k}}\right\}_{k=1}^{l}$. By adjusting the convex function $w=\left\{w_{m}\right\}_{m \in \Sigma_{p}(1)}$ by linear function, we may assume that $w_{m}=0$ for $m \in S_{\sigma}$ and $w_{m}>0$ for $m \in$ $\Sigma_{p}(1) \backslash S_{\sigma}$. Then, we have $s_{m}=t^{w_{m}} z^{m}$, where $m=\left\{m^{k}\right\}_{k=1}^{l}$ also denotes the coordinate of $m$ with respect to the basis $\left\{m_{k}\right\}_{k=1}^{l}$. It is easy to see that this coordinate $z$ is a special case of the toroidal coordinate $z$ defined in Section 2. Let

$$
\alpha_{p \sigma}=\frac{i}{\pi} \sum_{m \in S_{\sigma}} \frac{1}{a_{m}^{2}} \partial a_{m} \bar{\partial} a_{m}, \alpha_{t, p \sigma}=\left.\alpha_{p \sigma}\right|_{X_{t}} .
$$

\section{Proposition 4.4.}

$$
\alpha_{p \sigma} \leq \alpha \leq C(w) \alpha_{p \sigma}
$$

along $z$ direction in $U_{p \sigma}^{0}$. Consequently,

$$
C_{1}\left(\prod_{m \in S_{\sigma}} \frac{1}{a_{m}^{2}}\right) \hat{V}_{t} \leq \omega_{t}^{n} \leq C_{2}\left(\prod_{m \in S_{\sigma}} \frac{1}{a_{m}^{2}}\right) \hat{V}_{t}, \quad \text { in } \quad U_{p \sigma}^{0} \cap X_{t} .
$$


Proof. By the definition of $U_{p \sigma}^{0}$, clearly $\alpha_{p \sigma} \leq \alpha \leq C(w) \alpha_{p \sigma}$ along $z$ direction in $U_{p \sigma}^{0}$. Therefore, $\omega_{t} \sim \hat{\omega}_{t}+\alpha_{t} \sim \hat{\omega}_{t}+\alpha_{t, p \sigma}$ according to Lemma 4.2. Since $\alpha_{t, p \sigma}^{l+1}=0$. In $X_{t} \cap U_{p \sigma}^{0}$, we have

$$
\omega_{t}^{n} \sim\left(\hat{\omega}_{t}+\alpha_{t, p \sigma}\right)^{n} \sim \hat{\omega}_{t}^{n-l} \wedge \alpha_{t, p \sigma}^{l} .
$$

According to formula (3.1),

$$
\hat{V}_{t}=\rho(z)\left(\prod_{j=1}^{l} \frac{d z_{j} d \bar{z}_{j}}{\left|z_{j}\right|^{2}}\right)\left(\prod_{j=l+1}^{n} d z_{j} d \bar{z}_{j}\right) .
$$

Hence,

$$
\hat{\omega}_{t}^{n-l} \wedge \alpha_{t, p \sigma}^{l} \sim\left(\prod_{m \in S_{j}} \frac{\partial a_{m} \bar{\partial} a_{m}}{a_{m}^{2}}\right)\left(\prod_{j=l+1}^{n} d z_{j} d \bar{z}_{j}\right) \sim\left(\prod_{m \in S_{p}} \frac{1}{a_{m}^{2}}\right) \hat{V}_{t} .
$$

Notice that $V_{t}=h \hat{V}_{t}$ is the Kähler potential of $\omega_{t}$. Assume

$$
e^{-\phi_{t}}=\frac{\omega_{t}^{n}}{V_{t}}
$$

Proposition 4.5. $\left|\phi_{t}\right|$ is bounded independent of $t$.

Proof. According to Proposition 4.3, it is sufficient to verify in each $U_{p \sigma}^{0} \cap X_{t}$ for $p \in \Sigma$ and $\sigma \in \Sigma_{p}(l)$. Proposition 4.4 implies that

$$
\frac{\omega_{t}^{n}}{V_{t}} \sim \eta^{2\left|\Sigma_{p}(1)\right|} \sim 1 \text { in } U_{p \sigma}^{0} \cap X_{t} .
$$

Therefore, $\left|\phi_{t}\right|$ is bounded independent of $t$.

Let $g_{t}$ denote the Kähler metric corresponding to the Kähler form $\omega_{t}$, then we have

Proposition 4.6. The curvature of $g_{t}$ and its derivatives are all uniformly bounded with respect to $t$. 
Proof. On a Riemannian manifold $(M, g)$, we call a basis $\left\{v_{i}\right\}$ proper if the corresponding metric matrix satisfies $C_{1}\left(\delta_{i j}\right) \leq\left(g_{i j}\right) \leq C_{2}\left(\delta_{i j}\right)$ for $C_{1}, C_{2}>$ 0 . To verify that the curvature of the Riemannian metric $g$ and all its covariant derivatives are bounded, it is sufficient to find a proper basis $\left\{v_{i}\right\}$ satisfying that the coefficients of $\left[v_{i}, v_{j}\right]$ with respect to the basis $\left\{v_{i}\right\}$ and all their derivatives with respect to $\left\{v_{i}\right\}$ are bounded, such that $g_{i j}$ and all their derivatives with respect to $\left\{v_{i}\right\}$ are bounded.

According to Proposition 4.3, it is sufficient to verify in each $U_{p \sigma}^{0} \cap X_{t}$. Let $W_{j}=a_{m_{j}} z_{j} \frac{\partial}{\partial z_{j}}$ for $1 \leq j \leq l$ and $W_{j}=\frac{\partial}{\partial z_{j}}$ for $l+1 \leq j \leq n$. According to Proposition 4.4, it is straightforward to check that the basis $\left\{W_{j}, \bar{W}_{j}\right\}_{j=1}^{n}$ is proper in $U_{p \sigma}^{0} \cap X_{t}$. Namely, $C_{1}\left(\delta_{i j}\right) \leq\left(g_{i \bar{j}}\right) \leq C_{2}\left(\delta_{i j}\right)$ for some $C_{1}, C_{2}>0$, where $\left(g_{i \bar{j}}\right)$ denotes the metric matrix with respect to the basis $\left\{W_{j}, \bar{W}_{j}\right\}_{j=1}^{n}$. (For the upper bound estimate, we need $\frac{a_{m_{j}}}{a_{m}}$ to be bounded for $1 \leq j \leq l$ and $m \in \Sigma_{I}(1) \backslash S_{i}$, which is due to our restriction to $U_{p \sigma}^{0}$.)

For $1 \leq j \leq l,\left\|s_{m_{j}}\right\|_{m_{j}}^{2}=\rho_{j}\left|z_{j}\right|^{2}$.

$$
\begin{aligned}
& W_{k}\left(a_{m_{j}}\right)=\frac{W_{k}\left(\left\|s_{m_{j}}\right\|_{m_{j}}^{2}\right)}{\left\|s_{m_{j}}\right\|_{m_{j}}^{2}}=\frac{W_{k}\left(\rho_{j}\right)}{\rho_{j}}+\frac{W_{i}\left(\left|z_{j}\right|^{2}\right)}{\left|z_{j}\right|^{2}} . \\
& W_{k}\left(a_{m_{j}}\right)=a_{m_{k}}\left(z_{k} \frac{\partial \log \rho_{j}}{\partial z_{k}}+\delta_{k j}\right) \quad \text { for } 1 \leq k \leq l . \\
& W_{k}\left(a_{m_{j}}\right)=\frac{\partial \log \rho_{j}}{\partial z_{k}} \text { for } l+1 \leq k \leq n .
\end{aligned}
$$

The functions

$$
\begin{aligned}
& \frac{1}{a_{m_{j}}}, s_{m} P(a), \bar{s}_{m} P(a), z_{j} P\left(a_{m_{j}}\right), \bar{z}_{j} P\left(a_{m_{j}}\right), \\
& \frac{a_{m_{j}}}{a_{m}}, \frac{\log |t|^{2}}{a_{m}}, \frac{a_{m}}{\log |t|^{2}}, \text { for } m \in \Sigma_{p}(1) \backslash S_{\sigma}, 1 \leq j \leq l .
\end{aligned}
$$

are all bounded in $U_{p \sigma}^{0} \cap X_{t}$, where $P(a)$ is a polynomial on $\left(\left\{a_{m_{j}}\right\}_{j=1}^{l}, \log t\right)$ and $P\left(a_{m_{j}}\right)$ is a polynomial on $a_{m_{j}}$. The above computations imply that the derivatives of functions in (4.1) with respect to $\left\{W_{j}, \bar{W}_{j}\right\}_{j=1}^{n}$ are smooth functions of terms in (4.1) and other smooth bounded terms. Therefore, they are bounded.

It is straightforward to check that $g_{i \bar{j}}$ and the coefficients of $\left[W_{j}, W_{k}\right]$, $\left[W_{j}, \bar{W}_{k}\right],\left[\bar{W}_{j}, \bar{W}_{k}\right]$ with respect to the basis $\left\{W_{j}, \bar{W}_{j}\right\}_{j=1}^{n}$ are all smooth functions of terms in (4.1) and other bounded smooth terms. Consequently, any derivatives of theirs with respect to $\left\{W_{j}, \bar{W}_{j}\right\}_{j=1}^{n}$ are also smooth functions of terms in (4.1) and other bounded terms, therefore, are all bounded. 
Proposition 4.7. For any $k,\left\|\phi_{t}\right\|_{C^{k}, g_{t}}$ is uniformly bounded with respect to $t$.

Proof. Similar to the proof of the previous proposition, in $U_{p \sigma}^{0} \cap X_{t}$, it is straightforward to check according to Proposition 4.5 and the explicit expression of $\phi_{t}$ that $\phi_{t}$ is a bounded smooth function of terms in (4.1) and other smooth bounded terms. Consequently, all multi-derivatives of $\phi_{t}$ with respect to $\left\{W_{j}, \bar{W}_{j}\right\}_{j=1}^{n}$ are smooth functions of terms in (4.1) and other smooth bounded terms. Therefore, they are bounded.

\section{Construction of Kähler-Einstein metric via complex Monge-Ampère.}

In this section, we will use the same notions as in the previous sections. In [9], using the Monge-Ampère estimate of Aubin and Yau, Tian essentially proved the following.

Theorem 5.1. (Tian) Assume that $\phi_{t}$, the curvature of $g_{t}$ and their multiderivatives are all bounded uniformly independent of $t$, then the KählerEinstein metric $g_{E, t}$ on $X_{t}$ will converge to the complete Cheng-Yau KählerEinstein metric $g_{E, 0}$ on $X_{0} \backslash \operatorname{Sing}\left(X_{0}\right)$ in the sense of Cheeger-Gromov: there are an exhaustion of compact subsets $F_{\beta} \subset X_{0} \backslash \operatorname{Sing}\left(X_{0}\right)$ and diffeomorphisms $\psi_{\beta, t}$ from $F_{\beta}$ into $X_{t}$ satisfying:

(1) $X_{t} \backslash \bigcup_{\beta=1}^{\infty} \psi_{\beta, t}\left(F_{\beta}\right)$ consists of finite union of submanifolds of real codimension 1;

(2) for each fixed $\beta, \psi_{\beta, t}^{*} g_{E, t}$ converge to $g_{E, 0}$ on $F_{\beta}$ in $C^{k}$-topology on the space of Riemannian metrics as $t$ goes to 0 for any $k$.

Proof of Theorem 1.1. Proposition 2.3 reduces the theorem to the case that $\pi: \mathcal{X} \rightarrow B$ is simple, which is a direct corollary of Theorem 5.1 and Propositions 4.5, 4.6, and 4.7.

It is easy to see that our construction actually implies the following asymptotic description of the family of Kähler-Einstein metrics. 
Theorem 5.2. Kähler-Einstein metric $g_{E, t}$ on $X_{t}$ is uniformly quasiisometric to the explicit approximate metric $g_{t}$. More precisely, there exist constants $C_{1}, C_{2}>0$ independent of $t$ such that $C_{1} g_{t} \leq g_{E, t} \leq C_{2} g_{t}$.

Proof. The uniform $C^{0}$-estimate of the complex Monge-Ampère equations implies that $C_{1} \omega_{t}^{n} \leq \omega_{E, t}^{n} \leq C_{2} \omega_{t}^{n}$ for some $C_{1}, C_{2}>0$. The uniform $C^{2}$ estimate of the complex Monge-Ampère equations implies that $\operatorname{Tr}_{g_{t}} g_{E, t}$ is uniformly bounded from above. Combining these two estimates, we get our conclusion.

\section{Weil-Peterson metric near degeneration.}

In this section, we will start with the discussion of the toric case, which is of independent interest and the estimate is more precise. Then, we will proceed to the global toroidal case.

\subsection{The Toric case.}

Note: The notations in this subsection are the same as in Subsection 2.1. Unless specified otherwise, the notations in this section will not be carried over to other parts of this paper.

Example: Consider a toric degeneration $\pi: \mathcal{X} \rightarrow B \cong \mathbb{C}$ determined by a complete fan $\Sigma$ in $M$ and an integral piecewise linear convex function determined by $\left\{w_{m}\right\}_{m \in \Sigma(1)}$. For $i \in \Sigma(n)$, assume $w_{m}=0$ for $m \in S_{i}$ and $w_{m}>0$ for $m \in \Sigma(1) \backslash S_{i}$. With $S_{i}=\left\{m_{1}, \cdots, m_{n}\right\}$ and toric coordinate $z_{j}=s_{m_{j}}$ for $1 \leq j \leq n$, we have

$$
\omega=\frac{i}{\pi} \sum_{j=1}^{n} \frac{d z_{j} \wedge d \bar{z}_{j}}{\left|z_{j}\right|^{2}\left(\log \left|z_{j}\right|^{2}\right)^{2}}+\frac{i}{\pi} \sum_{m \in \Sigma(1) \backslash S_{i}} \frac{d s_{m} \wedge d \bar{s}_{m}}{\left|s_{m}\right|^{2}\left(\log \left|s_{m}\right|^{2}\right)^{2}} .
$$

Let

$$
W=\frac{\nabla \log t}{|\nabla \log t|^{2}}
$$

then $\left.\bar{\partial} W\right|_{X_{t}}$ is a natural representative of Kodaira-Spencer deformation class in the Dolbeaut cohomology $H^{1}\left(T_{X_{t}}\right)$. $W$ can also be determined by the conditions $\pi_{*} W=t \frac{d}{d t}$ and $\left.i(W) \omega\right|_{X_{t}}=0$ for all $t$. Let $a_{j}=\log \left|z_{j}\right|^{2}$ and $a_{m}=\log \left|s_{m}\right|^{2}$ for $m \in \Sigma(1) \backslash S_{i}$. We will use $\rho=1+O\left(a_{j} / a_{m}\right)$ to denote a bounded smooth function on $a_{j} / a_{m}$ for $1 \leq j \leq n, m \in \Sigma(1) \backslash S_{i}$. (Here, 
$O\left(a_{j} / a_{m}\right)$ is a shorthand for $O\left(a_{j} / a_{m}, 1 \leq j \leq n, m \in \Sigma(1) \backslash S_{i}\right)$.) It is straightforward to derive that

$$
\begin{aligned}
\omega_{t} & =\left.\omega\right|_{X_{t}}=\frac{i}{\pi} \sum_{j, k=1}^{n} g_{j \bar{k}} \frac{\partial a_{j}}{a_{j}} \wedge \frac{\bar{\partial} a_{k}}{a_{k}}, \\
g_{j \bar{k}} & =\delta_{j k}+a_{j} a_{k} O\left(\frac{1}{a_{m}^{2}}\right), \quad g^{j \bar{k}}=\delta_{j k}+a_{j} a_{k} O\left(\frac{1}{a_{m}^{2}}\right), \\
\omega_{t}^{n} & =n !\left(\frac{i}{\pi}\right)^{n} \rho \prod_{j=1}^{n} \frac{d z_{j} \wedge d \overline{z_{j}}}{a_{j}^{2}\left|z_{j}\right|^{2}}=n !\left(\frac{1}{\pi}\right)^{n} \rho \prod_{j=1}^{n} \frac{d a_{j} \wedge d \theta_{j}}{a_{j}^{2}} .
\end{aligned}
$$

\section{Lemma 6.1.}

$$
W=t \frac{\partial}{\partial t}-\sum_{j=1}^{n} \sum_{m \in \Sigma(1) \backslash S_{i}} w_{m} m^{j} \frac{a_{j}^{2}}{a_{m}^{2}} \rho z_{j} \frac{\partial}{\partial z_{j}} .
$$

Proof. Since $\pi_{*} W=t \frac{d}{d t}$, we may assume that $W=t \frac{\partial}{\partial t}+\sum_{j=1}^{n} q_{j} z_{j} \frac{\partial}{\partial z_{j}}$. $\left.i(W) \omega\right|_{X_{t}}=0$ implies that

$$
\sum_{m \in \Sigma(1) \backslash S_{i}} w_{m} \frac{d \bar{s}_{m}}{\bar{s}_{m} a_{m}^{2}}+\sum_{j=1}^{n} q_{j} \frac{d \bar{z}_{j}}{\bar{z}_{j} a_{j}^{2}}+\sum_{m \in \Sigma(1) \backslash S_{i}} \sum_{j=1}^{n} q_{j} m^{j} \frac{d \bar{s}_{m}}{\bar{s}_{m} a_{m}^{2}}=0 .
$$

Consequently, $q_{j}=-\sum_{m \in \Sigma(1) \backslash S_{i}} w_{m} m^{j} \frac{a_{j}^{2}}{a_{m}^{2}} \rho$.

Define $F=a=\left(a_{1}, \cdots, a_{n}\right): \mathcal{X} \rightarrow \mathbb{R}^{n}$. Let $A_{i}(x)=\min _{m \in \Sigma(1) \backslash S_{i}} a_{m}(x)$. For $\eta>0$, consider the domain $U_{i, \eta}=\left\{x \in U_{\eta} \mid A_{i}(x) \geq A_{i^{\prime}}(x)\right.$ for $\left.i^{\prime} \in \Sigma(n)\right\}$, where $U_{\eta}=\left\{x \in \mathcal{X} \mid a_{m}(x) \geq \eta\right.$ for $\left.m \in \Sigma(1)\right\}$. Notice that Proposition 4.1 implies that $A_{i}(x) \geq \lambda_{1} \tau>0$ for $x \in U_{i, \eta}$. It is easy to observe that there exist $c^{\prime}>c>0$ such that $[\eta, c \tau]^{n} \subset F\left(X_{t} \cap U_{i, \eta}\right) \subset\left[\eta, c^{\prime} \tau\right]^{n}$. For $\omega_{t}$ and $W$ as in the previous example, we have

Proposition 6.2. There exists a constant $C_{i, \eta} \geq 0$, such that

$$
\frac{\int_{X_{t} \cap U_{i, \eta}}\|\bar{\partial} W\|^{2} \omega_{t}^{n}}{\int_{X_{t} \cap U_{i, \eta}} \omega_{t}^{n}}=\frac{C_{i, \eta}+O\left(\tau^{-1} \log \tau\right)}{\left.\left.|\log | t\right|^{2}\right|^{3}} .
$$


Proof. We may compute the volume of $X_{t} \cap U_{i, \eta}$.

$$
\begin{aligned}
\int_{X_{t} \cap U_{i, \eta}} \omega_{t}^{n} & =n ! 2^{n} \int_{F\left(X_{t} \cap U_{i, \eta}\right)} \rho \prod_{j=1}^{n} \frac{d a_{j}}{a_{j}^{2}}=n ! 2^{n} \int_{[\eta, c \tau]^{n}} \rho \prod_{j=1}^{n} \frac{d a_{j}}{a_{j}^{2}}(1+O(1 / \tau)) \\
& =n ! 2^{n} \prod_{j=1}^{n}\left(\int_{\eta}^{c \tau} \frac{d a_{j}}{a_{j}^{2}}\right)\left(1+O\left(\tau^{-1} \log \tau\right)\right)=\frac{n ! 2^{n}}{\eta^{n}}\left(1+O\left(\tau^{-1} \log \tau\right)\right) .
\end{aligned}
$$

Notice

$$
\bar{\partial}\left(\frac{a_{j}^{2}}{a_{m}^{2}} \rho\right)=\rho \frac{2 a_{j}}{a_{m}^{2}} \bar{\partial} a_{j}+\frac{a_{j}^{2}}{a_{m}^{2}} O\left(\frac{\bar{\partial} a_{j^{\prime}}}{a_{m}}\right) .
$$

It is straightforward to compute

$$
\left\|\sum_{m \in \Sigma(1) \backslash S_{i}} w_{m} m^{j} \bar{\partial}\left(\frac{a_{j}^{2}}{a_{m}^{2}} \rho\right)\right\|^{2}=4 a_{j}^{4} \rho\left|\sum_{m \in \Sigma(1) \backslash S_{i}} \frac{w_{m} m^{j}}{a_{m}^{2}}\right|^{2} .
$$

According to Lemma 6.1, we have

$$
\begin{aligned}
\bar{\partial} W & =-\sum_{j=1}^{n} \sum_{m \in \Sigma(1) \backslash S_{i}} w_{m} m^{j} \bar{\partial}\left(\frac{a_{j}^{2}}{\left.a_{m}^{2} \rho\right) z_{j} \frac{\partial}{\partial z_{j}}}\right. \\
\|\bar{\partial} W\|^{2} & =\sum_{j=1}^{n} 4 a_{j}^{2} \rho\left|\sum_{m \in \Sigma(1) \backslash S_{i}} \frac{w_{m} m^{j}}{a_{m}^{2}}\right|^{2} \cdot \\
\int_{X_{t} \cap U_{i, \eta}}\|\bar{\partial} W\|^{2} \omega_{t}^{n} & =n ! 2^{n} \int_{F\left(X_{t} \cap U_{i, \eta}\right)} \sum_{j=1}^{n} 4 \rho\left|\sum_{m \in \Sigma(1) \backslash S_{i}} \frac{w_{m} m^{j}}{a_{m}^{2}}\right|^{2} d a_{j} \prod_{j^{\prime} \neq j} \frac{d a_{j^{\prime}}}{a_{j^{\prime}}^{2}}
\end{aligned}
$$

For each $j$, let

$\tilde{U}_{i j, \eta}^{0}=\left\{a \in \mathbb{R}^{n} \mid \eta \leq a_{j} \leq c_{j} \tau, \eta \leq a_{j^{\prime}} \leq c \tau\right.$, for $\left.j^{\prime} \neq j\right\}$.

$\tilde{U}_{i j, \eta}^{1}=\left\{a \in F\left(X_{t} \cap U_{i, \eta}\right) \mid \eta \leq a_{j^{\prime}} \leq c \tau\right.$, for $\left.j^{\prime} \neq j\right\}, \tilde{U}_{i j, \eta}^{2}=F\left(X_{t} \cap U_{i, \eta}\right) \backslash \tilde{U}_{i j, \eta}^{1}$. 
It is straightforward to derive that

$$
\begin{aligned}
& \int_{\tilde{U}_{i j, \eta}^{2}} 4 \rho\left|\sum_{m \in \Sigma(1) \backslash S_{i}} \frac{w_{m} m^{j}}{a_{m}^{2}}\right|^{2} d a_{j} \prod_{j^{\prime} \neq j} \frac{d a_{j^{\prime}}}{a_{j^{\prime}}^{2}} \\
& =O\left(\frac{1}{\eta^{n-1} \tau^{4}}\right) \int_{\tilde{U}_{i j, \eta}^{1}} 4 \rho\left|\sum_{m \in \Sigma(1) \backslash S_{i}} \frac{w_{m} m^{j}}{a_{m}^{2}}\right|^{2} d a_{j} \prod_{j^{\prime} \neq j} \frac{d a_{j^{\prime}}}{a_{j^{\prime}}^{2}} \\
& =\int_{\tilde{U}_{i j, \eta}^{1}} 4 \rho_{j}\left(b_{j}\right)\left|\sum_{m \in \Sigma(1) \backslash S_{i}} \frac{w_{m} m^{j}}{\left(w_{m} \tau+m^{j} a_{j}\right)^{2}}\right|^{2} d a_{j} \prod_{j^{\prime} \neq j} \frac{d a_{j^{\prime}}}{a_{j^{\prime}}^{2}}+O\left(\frac{\log \tau}{\eta^{n-1} \tau^{4}}\right) \\
& \left(\int_{\tilde{U}_{i j, \eta}^{1}}-\int_{\tilde{U}_{i j, \eta}^{0}}\right) 4 \rho_{j}\left(b_{j}\right)\left|\sum_{m \in \Sigma(1) \backslash S_{i}} \frac{w_{m} m^{j}}{\left(w_{m} \tau+m^{j} a_{j}\right)^{2}}\right|^{2} d a_{j} \prod_{j^{\prime} \neq j} \frac{d a_{j^{\prime}}}{a_{j^{\prime}}^{2}} \\
& =\left.\left.O\left(\frac{\log \tau}{\eta^{n-1} \tau^{4}}\right) \int_{\tilde{U}_{i j, \eta}^{0}} 4 \rho_{j}\left(b_{j}\right)\right|_{m \in \Sigma(1) \backslash S_{i}} \frac{w_{m} m^{j}}{\left(w_{m} \tau+m^{j} a_{j}\right)^{2}}\right|^{2} d a_{j} \prod_{j^{\prime} \neq j} \frac{d a_{j^{\prime}}}{a_{j^{\prime}}^{2}} \\
& =\frac{4}{\left.\left.\eta^{n-1}|\log | t\right|^{2}\right|^{3}} \sum_{j=1}^{n} B_{j} \prod_{j^{\prime} \neq j} \int_{1}^{+\infty} \frac{d x_{j^{\prime}}}{x_{j^{\prime}}^{2}}+O\left(\frac{\log \tau}{\eta^{n-1} \tau^{4}}\right),
\end{aligned}
$$

where

$$
B_{j}=\int_{0}^{c_{j}} \rho_{j}\left(b_{j}\right)\left|\sum_{m \in \Sigma(1) \backslash S_{i}} \frac{w_{m} m^{j}}{\left(w_{m}+m^{j} b_{j}\right)^{2}}\right|^{2} d b_{j},
$$

with $c_{j}=\frac{w_{\tilde{m}_{j}}}{1-\tilde{m}_{j}^{j}}, b_{j}=a_{j} / \tau, x_{j^{\prime}}=a_{j^{\prime}} / \eta$, and $\rho_{j}\left(b_{j}\right)$ is $\rho$ replacing $a_{j} / a_{m}$ by $b_{j} /\left(w_{m}+m^{j} b_{j}\right)$ and replacing $a_{j^{\prime}}$ for $j^{\prime} \neq j$ by zero. Combining all these estimates, we have

$$
\int_{X_{t} \cap U_{i, \eta}}\|\bar{\partial} W\|^{2} \omega_{t}^{n}=\frac{n ! 2^{n+2}}{\eta^{n-1}} \frac{1}{\left.\left.|\log | t\right|^{2}\right|^{3}} \sum_{j=1}^{n}\left(B_{j}+O\left(\tau^{-1} \log \tau\right)\right),
$$

We may take $C_{i, \eta}=4 \eta \sum_{j=1}^{n} B_{j}$ for the proposition to hold.

\subsection{The Toroidal case.}

With respect to the local Kähler metric $\omega_{p}=\hat{\omega}+\frac{i}{2 \pi} \partial \bar{\partial} \log h_{p}$ and parameterizing function $t$ on $U_{p}$, we can similarly define $W_{(p)}=\frac{\nabla \log t}{|\nabla \log t|^{2}}$. Let 
$W=\sum_{p \in \Sigma} \mu_{p} W_{(p)} . \bar{\partial} W$ also represents the Kodaira-Spencer deformation class. We have

Proposition 6.3. There exists a constant $C>0$ independent of $t$ such that

$$
\int_{X_{t}}\|\bar{\partial} W\|_{g_{t}}^{2} \omega_{t}^{n} \leq \frac{C}{\left.\left.|\log | t\right|^{2}\right|^{3}} \int_{X_{t}} \omega_{t}^{n}
$$

Proof. Locally in each $U_{q \sigma}^{0}$, we will use similar coordinate and proper basis $\left\{W_{j}, \bar{W}_{j}\right\}_{j=1}^{n}$ as in the proof of Proposition 4.6. Then the dual basis is $\left\{\beta_{j}, \bar{\beta}_{j}\right\}_{j=1}^{n}$, where $\beta_{j}=\frac{d z_{j}}{a_{j} z_{j}}, a_{j}=\log \left|z_{j}\right|^{2}$ for $1 \leq j \leq l$ and $\beta_{i}=d z_{i}$ for $l+1 \leq j \leq n$. Recall that $O(1)$ denotes a smooth function on terms in (4.1) and other smooth bounded terms. (Notice that here we assume $a_{m}=\log \left|s_{m}\right|^{2}$, which is slightly different from (4.1) and do not affect our arguments here. In this proof, we are using $a_{j}$ to denote $a_{m_{j}}$ and $O\left(a_{j} / a_{m}\right)$ as a shorthand for $O\left(a_{j} / a_{m}, 1 \leq j \leq n, m \in \Sigma_{q}(1) \backslash S_{\sigma}\right)$.) We will also use $O(1)$ to denote a tensor with $O(1)$ coefficients with respect to the proper and dual proper basis. It is easy to see that the action of the proper basis $\left\{W_{j}, \bar{W}_{j}\right\}_{j=1}^{n}$ will send $O(1)$ to $O(1)$, also $\bar{\partial} W_{j}=O(1)$. Under such notation, we have

$$
\begin{aligned}
\omega_{t, q} & =\sum_{j, k=1}^{n} g_{j \bar{k}} \beta_{j} \bar{\beta}_{k} . \\
g_{j \bar{k}} & =\delta_{j k}\left(1+\frac{1}{a_{k}} O(1)\right)+a_{j} a_{k} O\left(\frac{1}{a_{m}^{2}}\right)+\frac{1}{a_{j} a_{k}} O(1), \text { for } 1 \leq j, k \leq l . \\
g_{j \bar{k}} & =\frac{1}{a_{k}} O(1), g^{j \bar{k}}=\frac{1}{a_{k}} O(1), \text { for } 1 \leq k \leq l \text { and } l+1 \leq j \leq n .
\end{aligned}
$$

It is straightforward to derive that

$$
\begin{aligned}
\left.i\left(t \frac{\partial}{\partial t}\right) \omega_{q}\right|_{X_{t}} & =\sum_{j=1}^{l} \sum_{m \in \Sigma_{q}(1) \backslash S_{\sigma}} w_{m} m^{j} \frac{a_{j}}{a_{m}^{2}} \bar{\beta}_{j}+O\left(\frac{1}{a_{m}^{2}}\right), \\
W_{(q)} & =t \frac{\partial}{\partial t}-\sum_{j=1}^{l} \sum_{m \in \Sigma_{q}(1) \backslash S_{\sigma}} w_{m} m^{j} \frac{a_{j}}{a_{m}^{2}} \rho W_{j}+O\left(\frac{1}{a_{m}^{2}}\right), \\
\bar{\partial} W_{(q)} & =-\sum_{j=1}^{l} \sum_{m \in \Sigma_{q}(1) \backslash S_{\sigma}} w_{m} m^{j} \bar{\partial}\left(\frac{a_{j}^{2}}{a_{m}^{2}} \rho\right) z_{j} \frac{\partial}{\partial z_{j}}+O\left(\frac{1}{a_{m}^{2}}\right) .
\end{aligned}
$$


Applying Proposition 6.2, we can find $C>0$ independent of $t$ such that

$$
\int_{U_{q \sigma}^{0} \cap X_{t}}\left\|\bar{\partial} W_{(q)}\right\|_{g_{t}}^{2} \omega_{t}^{n} \leq \frac{C}{\left.\left.|\log | t\right|^{2}\right|^{3}} \int_{U_{q \sigma}^{0} \cap X_{t}} \omega_{t}^{n} .
$$

The rest of the proof closely resembles the proof of Lemma 4.2. For any $x \in X_{t}$, there exist a $q \in \Sigma$ such that $x \in X_{t} \cap U_{q}^{0}$. Since

$$
\sum_{p \in \Sigma} \mu_{p}=1, \quad \sum_{p \in \Sigma} \bar{\partial} \mu_{p}=0
$$

We have

$$
\sum_{p \in \Sigma} \bar{\partial} \mu_{p} W_{(p)}=\sum_{p \in \Sigma} \bar{\partial} \mu_{p}\left(W_{(p)}-W_{(q)}\right)
$$

Since $U_{q}^{0} \cap \operatorname{supp}\left(\mu_{p}\right)=\emptyset$ when $D_{q} \not \subset \bar{D}_{p}$ according to (2.3), we may consider only those $p \in \Sigma$ satisfying $D_{q} \subset \bar{D}_{p}$. As in the proof of Lemma 4.2, for such $p, q \in \Sigma$, we can naturally define $\Sigma_{q}^{q} \cap \Sigma_{p}^{p}$. For any $m \in \Sigma_{p}^{p} \backslash \Sigma_{q}^{q},\left(s_{m}\right) \cap \bar{D}_{q}=\emptyset$. Consequently, $\left\|s_{m}\right\|_{m}^{2}=1$ and $a_{m}=\eta$ on $\tilde{U}_{q}$ for $m \in \Sigma_{p}^{p} \backslash \Sigma_{q}^{q}$. Hence

$$
\begin{gathered}
W_{(p)}-W_{(q)}=\sum_{j=1}^{l} O\left(\frac{a_{j}}{a_{m}^{2}}\right) W_{j}+O\left(\frac{1}{a_{m}^{2}}\right) \\
\bar{\partial} W_{(p)}=\sum_{j=1}^{l} O\left(\frac{a_{j}}{a_{m}^{2}}\right)+O\left(\frac{1}{a_{m}^{2}}\right)
\end{gathered}
$$

on $\operatorname{supp}\left(\mu_{p}\right) \cap U_{q} \subset U_{p} \cap U_{q}$. From the explicit expressions of $\mu_{p}$, it is straightforward to check that $\bar{\partial} \mu_{p}=O(1 / \log \tau)$ with respect to the Hermitian metric $\omega_{t}$. Consequently,

$$
\begin{aligned}
& \int_{U_{q \sigma}^{0} \cap X_{t}}\left\|\sum_{p \in \Sigma} \bar{\partial} \mu_{p} W_{(p)}\right\|_{g_{t}}^{2} \omega_{t}^{n} \leq \frac{C}{\tau^{3} \log \tau} \int_{U_{q \sigma}^{0} \cap X_{t}} \omega_{t}^{n} . \\
& \int_{U_{q \sigma}^{0} \cap X_{t}}\left\|\sum_{p \in \Sigma} \mu_{p} \bar{\partial} W_{(p)}\right\|_{g_{t}}^{2} \omega_{t}^{n} \leq \frac{C}{\tau^{3}} \int_{U_{q \sigma}^{0} \cap X_{t}} \omega_{t}^{n} .
\end{aligned}
$$

Combine these estimates for all $\sigma \in \Sigma_{q}(l), q \in \Sigma$ applying to

$$
\bar{\partial} W=\sum_{p \in \Sigma} \mu_{p} \bar{\partial} W_{(p)}+\sum_{p \in \Sigma} \bar{\partial} \mu_{p} W_{(p)}
$$


we will get the desired estimate.

Remark: It is not hard to observe that the constant $C_{i, \eta} \geq 0$ in Proposition 6.2 is actually positive. With this observation and a bit more argument, one can show that the lower bound estimate in Proposition 6.3 (more precisely the estimate in Proposition 6.3 with the reversed inequality) is also true. Since such more precise estimates are not needed for arguments in this paper, we will omit them here.

Proof of Theorem 1.2. As pointed out in [9],

$$
\left.g_{W P}\left(\frac{d}{d t}, \frac{d}{d t}\right)\right|_{X_{t}}=\int_{X_{t}}\left\|H\left(\frac{d}{d t}\right)\right\|_{g_{E, t}}^{2} \omega_{E, t}^{n},
$$

where $H\left(\frac{d}{d t}\right)$ denote the harmonic representative of the Kodaira-Spencer deformation class. As mentioned earlier, such class can also be represented by $\frac{\bar{\partial} W}{t}$. Applying Proposition 6.3 and Theorem 5.2, we have

$$
\begin{aligned}
& \int_{X_{t}}\left\|H\left(\frac{d}{d t}\right)\right\|_{g_{E, t}}^{2} \omega_{E, t}^{n} \leq \int_{X_{t}}\left\|\frac{\bar{\partial} W}{t}\right\|_{g_{E, t}}^{2} \omega_{E, t}^{n} \leq C \int_{X_{t}}\left\|\frac{\bar{\partial} W}{t}\right\|_{g_{t}}^{2} \omega_{t}^{n} \\
& \quad \leq \frac{C}{|\log | t||^{3}|t|^{2}} .
\end{aligned}
$$

\section{References.}

[1] T. Aubin, Equation du type de Monge-Ampère sur les variétés Kähleriennes compacts, C. R. Acad. Sci. Prais 283 (1976), 119-121.

[2] J. Cheeger and M. Gromov, Collapsing Riemannian manifolds while keeping their curvature bounded. I, J. Differential Geom. 23 (1986), 309-346; II, J. Differential Geom. 32 (1990), 269-298.

[3] S. Y. Cheng and S.-T. Yau, On Inequality between Chern numbers of singular Kähler surfaces and characterization of orbit space of discrete group of $S U(2,1)$, Contemporary Math. 49 (1986), 31-43.

[4] N. Leung and P. Lu, Degeneration of Kähler Einstein metrics on complete Kähler manifolds, Comm. Analysis and Geom. 7 (1999), 431-449. 
[5] G. Kempf, F. Knudsen, D. Mumford and B. Saint-Donat, Toroidal Embeddings I, Lecture Notes in Mathematics 339, Springer-Verlag 1973.

[6] W. D. Ruan, On the convergence and collapsing of Kähler manifolds, Journal of Differential Geometry, 52 (1999), 1-40.

[7] W. D. Ruan, Canonical coordinates and Bergmann metrics, Communications in Analysis and Geometry, 6 (1998), 589-631.

[8] W. D. Ruan, Degeneration of Kähler-Einstein manifolds I: The normal crossing case, To appear in Comm. Contemporary Math.

[9] G. Tian, Degeneration of Kähler-Einstein manifolds I, Proceedings of Symposia in Pure Mathematics, 54(2), 595-609.

[10] G. Tian and S.-T. Yau, existence of Kähler-Einstein metrics on complete Kähler manifolds and their applications to algebraic geometry, Math. Aspects of String Theory (Edited by S. -T. Yau) pp. 574-628, World Sci. Publishing, 1987.

[11] S.-T. Yau, On Calabi's conjecture and some new results in algebraic geometry, Proc. Nat. Acad. Sci. U.S.A. 74 (1977), 1798-1799.

[12] S.-T. Yau, Métriques de Kähler-Einstein sur les variétés overtes, Astérisque 58 (1978), 163-167.

[13] S.-T. Yau, On the Ricci curvature of a compact Kähler manifold and the complex Monge-Ampère equation, I, Comm. Pure. and Appl. Math., 31 (1978), 339-411.

Department of Mathematics

UNIVERSiTy OF ILLINOIS AT CHICAGO

Chicago, IL 60607

Received August 29, 2003. 